\title{
Posttranscriptional control of NLRP3 inflammasome activation in colonic macrophages
}

\author{
AA Filardy ${ }^{1}, \mathrm{~J} \mathrm{He}^{1}, \mathrm{~J} \mathrm{Bennink}^{2}, \mathrm{~J} \mathrm{Yewdell}^{2}$ and BL Kelsall ${ }^{1}$
}

Colonic macrophages (cMPs) are important for intestinal homeostasis as they kill microbes and yet produce regulatory cytokines. Activity of the NLRP3 (nucleotide-binding leucine-rich repeat-containing pyrin receptor 3) inflammasome, a major sensor of stress and microorganisms that results in pro-inflammatory cytokine production and cell death, must be tightly controlled in the intestine. We demonstrate that resident cMPs are hyporesponsive to NLRP3 inflammasome

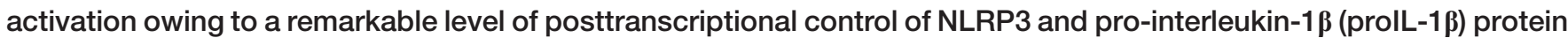
expression, which was also seen for tumor necrosis factor- $\alpha$ and IL-6, but lost during experimental colitis. Resident CMPs rapidly degraded NLRP3 and prolL-1 $\beta$ proteins by the ubiquitin/proteasome system. Finally, blocking IL-10Rsignaling in vivo enhanced NLRP3 and prolL-1 $\beta$ protein but not mRNA levels in resident CMPs, implicating a role for IL-10 in environmental conditioning of cMPs. These data are the first to show dramatic posttranscriptional control of inflammatory cytokine production by a relevant tissue-derived macrophage population and proteasomal degradation of prolL-1 $\beta$ and NLRP3 as a mechanism to control inflammasome activation, findings which have broad implications for our understanding of intestinal and systemic inflammatory diseases.

\section{INTRODUCTION}

The immune system in the intestine must maintain a symbiotic relationship with commensal bacteria while allowing host-defense against pathogens. Indeed, abnormal immune responses to commensal bacteria that occur in genetically susceptible hosts results in intestinal bowel disease. Intestinal macrophages are essential for maintaining immune homeostasis because of their efficient killing of invading bacteria and production of regulatory cytokines, which, in turn, support the expansion and/or survival of regulatory T-cell populations and inhibit the infiltration and activation of inflammatory cells. As was recently shown in mice, circulating monocytes differentiate into predominantly colonic macrophages (cMPs) that produce high levels of inhibitory cytokines in the steady-state but pro-inflammatory molecules during inflammation. ${ }^{1-4}$ The environmental factors that control the differentiation of monocytes into regulatory cMPs or inflammatory monocytes and cMPs are not yet clear, although interleukin (IL)-10 signaling appears to have a major role. ${ }^{5,6}$ Furthermore, the mechanisms that control the activation of pro-inflammatory pathways by intestinal macrophages are poorly understood, and studies to date have focused largely on transcriptional regulation by identifying gene-specific transcription factors, as well as enhancer landscapes, in macrophages from the small intestine and colon. ${ }^{7}$

Recent evidence indicates that inflammasome activation needs to be tightly controlled in the intestine. NLRP3 (NLR family, pyrin domain containing 3 ) and NLRC4 (NLR family, CARD domain containing 4) inflammasomes are multicomponent intracellular pattern-recognition receptors from the nucleotide-binding leucine rich repeat containing (NLR) family of proteins. ${ }^{8}$ Two signals are required for inflammasome-induced IL-1 $\beta$ secretion: one signal from Toll-like receptors (TLRs) or other pattern-recognition receptors that results in nuclear factor- $\mathrm{\kappa B}$-dependent transcription and translation of proIL-1 $\beta$ and NLRP3 but not NLRC4, which is constitutively expressed; and a second signal that triggers oligomerization of NLRP3 or NLRC4, together with the adaptor protein ASC and procaspase-1 leading to caspase-1 activation and IL-1 $\beta$ cleavage and release from proIL-1 $\beta$. NLRP3 is activated by numerous host and pathogen-derived factors indicative of stress and injury such as extracellular ATP,

${ }^{1}$ Mucosal Immunobiology Section, Laboratory of Molecular Immunology, NIAID, NIH, Bethesda, Maryland, USA and ${ }^{2}$ Cellular Biology and Viral Immunology Sections, Laboratory of Viral Diseases, NIAID, NIH, Bethesda, Maryland, USA. Correspondence: BL Kelsall (bkelsall@niaid.nih.gov)

Received 1 April 2015; accepted 29 September 2015; published online 2 December 2015. doi:10.1038/mi.2015.109 


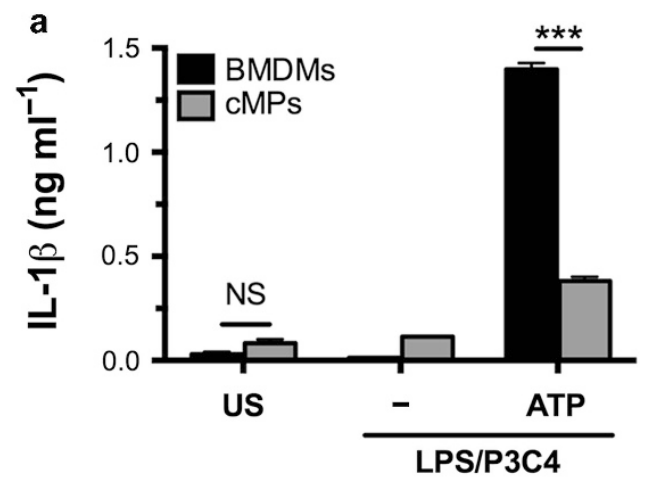

b

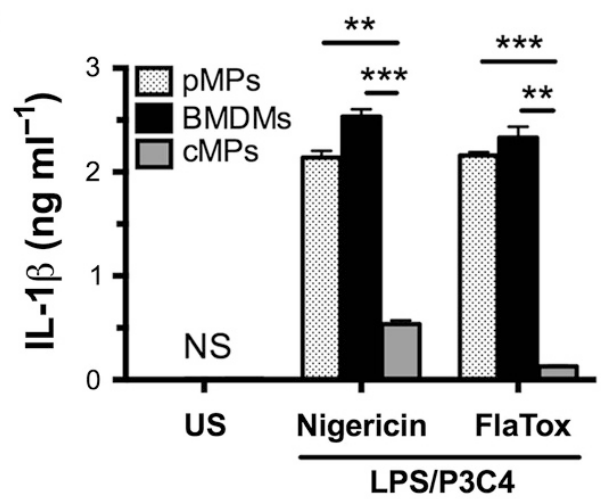

Figure 1 Resident colonic macrophages (cMPs) have poor NLRP3 (nucleotide-binding leucine-rich repeat-containing pyrin receptor 3 ) inflammasome activity. Resident cMPs, bone marrow-derived macrophages (BMDMs), and peritoneal macrophages (pMPs) were treated with lipopolysaccharide (LPS; $\left.200 \mathrm{ng} \mathrm{ml}^{-1}\right)$ and P3C4 $\left(2 \mu \mathrm{g} \mathrm{ml}^{-1}\right)$ for $4 \mathrm{~h}$ with and without stimulation for $1 \mathrm{~h}$ with ATP $(5 \mathrm{~mm})$, nigericin $(10 \mu \mathrm{m})$, or FlaTox $\left(3 \mu \mathrm{g} \mathrm{ml}^{-1}\right.$ of LFn-FlaA and $6 \mu \mathrm{g} \mathrm{ml}^{-1}$ of protective antigen). (a, b) Interleukin (IL)-1 $\beta$ measured in culture supernatants by enzyme-linked immunosorbent assay. Data are from sorted cells pooled from 30 colons presented as the mean \pm s.d. of two biological replicates and analyzed using an un-paired, two-tailed Student's $t$-test. ${ }^{* *} P<0.01$; ${ }^{\star * *} P<0.001$; NS, not significant, $P>0.05$. The data presented are representative of at least three independent experiments with similar results. US, unstimulated.

while NLRC4 is activated by intracellular products from pathogenic microbes, including flagellin, together with NAIPs (NLR family, apoptosis inhibitory proteins). Both NLRP3 and NLRC4 inflammasome activation, as well as IL-1 $\beta$ and IL-18, are important for colitis development in mouse models of intestinal bowel diseases. ${ }^{9,10}$ Furthermore, in patients with Crohn's disease, levels of IL-1 $\beta$ and IL-18 are highly elevated in inflamed tissues; ${ }^{11-14}$ however, the mechanisms by which inflammasomes are regulated in the intestine are incompletely understood.

Here we found that resident cMPs are hyporesponsive to NLRP3 inflammasome activation owing to a remarkable level of posttranscriptional control of NLRP 3 and proIL- $1 \beta$ protein expression. In homeostatic conditions, resident cMPs expressed high levels of mRNA but low levels of protein for NLRP 3 and proIL- $1 \beta$ both before and after exposure to TLR agonists and were hyporesponsive to NLRP3 activation. This posttranscriptional control was lost during experimental colitis where cMPs expressed high NLRP3 inflammasome activity. These results show that micro-environmental signals present in steady state, but not inflammatory conditions, orchestrate posttranscriptional control of inflammasome activation in resident cMPs. Furthermore, we demonstrated that resident cMPs rapidly degraded NLRP 3 and proIL- $1 \beta$ proteins by the ubiquitin/proteasome system and provided evidence supporting a role for IL-10 in this process. These findings demonstrate a new level of control of cMP function important for maintaining immunological homeostasis and have broad implications for our understanding of intestinal and systemic inflammatory diseases.

\section{RESULTS}

Resident cMPs are hyporesponsive to NLRP3 and NLRC4 inflammasome activation

To address NLRP3 inflammasome function, resident cMPs were purified by flow cytometry based on the expression of major histocompatibility complex (MHC) II, F4/80, and CD64 (see Supplementary Figure S1a online). cMPs, bone marrowderived macrophages (BMDMs), and peritoneal macrophages (pMPs) were treated with lipopolysaccharide (LPS; TLR4 agonist) and Pam3Cys-Ser-(Lys)4 (P3C4;TLR1/2 agonist) for $4 \mathrm{~h}$ followed by activation for $1 \mathrm{~h}$ with ATP or nigericin. We found that resident cMPs produced markedly less IL- $1 \beta$ when compared with either BMDMs or pMPs following TLR treatment and NLRP3 activation (Figure 1a,b). Immunoblotting for mature IL-1 $\beta$ confirmed poor activation of NLRP3 in resident cMPs (see Supplementary Figure S1b). We chose the combination of two common TLR agonists as it is likely that cMPs are exposed to multiple bacterial ligands in the colon, and because prior studies suggested cMPs respond poorly to LPS. ${ }^{15,16}$ We confirmed that adding P3C4 had no untoward effects on inflammasome induction or activation as both LPS or LPS/P3C4 and ATP induced similar levels of IL- $1 \beta$ secretion from cMPs and BMDMs (see Supplementary Figure S1c). Finally, to determine the NLR specificity of these effects, we also measured IL-1 $\beta$ production following the activation of NLRC4. Similar to NLRP3, cMPs but not BMDMs produced low levels of IL-1 $\beta$ following NLRC4 activation (Figure $\mathbf{1 b}$ and see Supplementary Figure S1b).

\section{NLRP3 and prolL-1 $\beta$ protein expression are posttranscriptionally regulated in resident cMPs}

We next addressed the possibility that poor NLRP3 inflammasome activity in resident cMPs was due to decreased TLR signaling, ${ }^{15,16}$ resulting in poor transcription of NLRP3 and IL-1 $\beta$ genes. Consistent with published data, ${ }^{17}$ mRNA for both NLRP3 and proIL-1 $\beta$ was undetectable in unstimulated BMDMs; however, we found very high levels of mRNA for NLRP3 and proIL-1 $\beta$ in unstimulated resident cMPs. In contrast, mRNA for ASC was expressed at similar levels in both BMDMs and cMPs (Figure 2a). TLR stimulation further enhanced the expression of mRNA for proIL- $1 \beta$ and NLRP3 in resident cMPs to levels similar to or higher than those induced in BMDMs, respectively (Figure 2a). Therefore, low NLRP3 

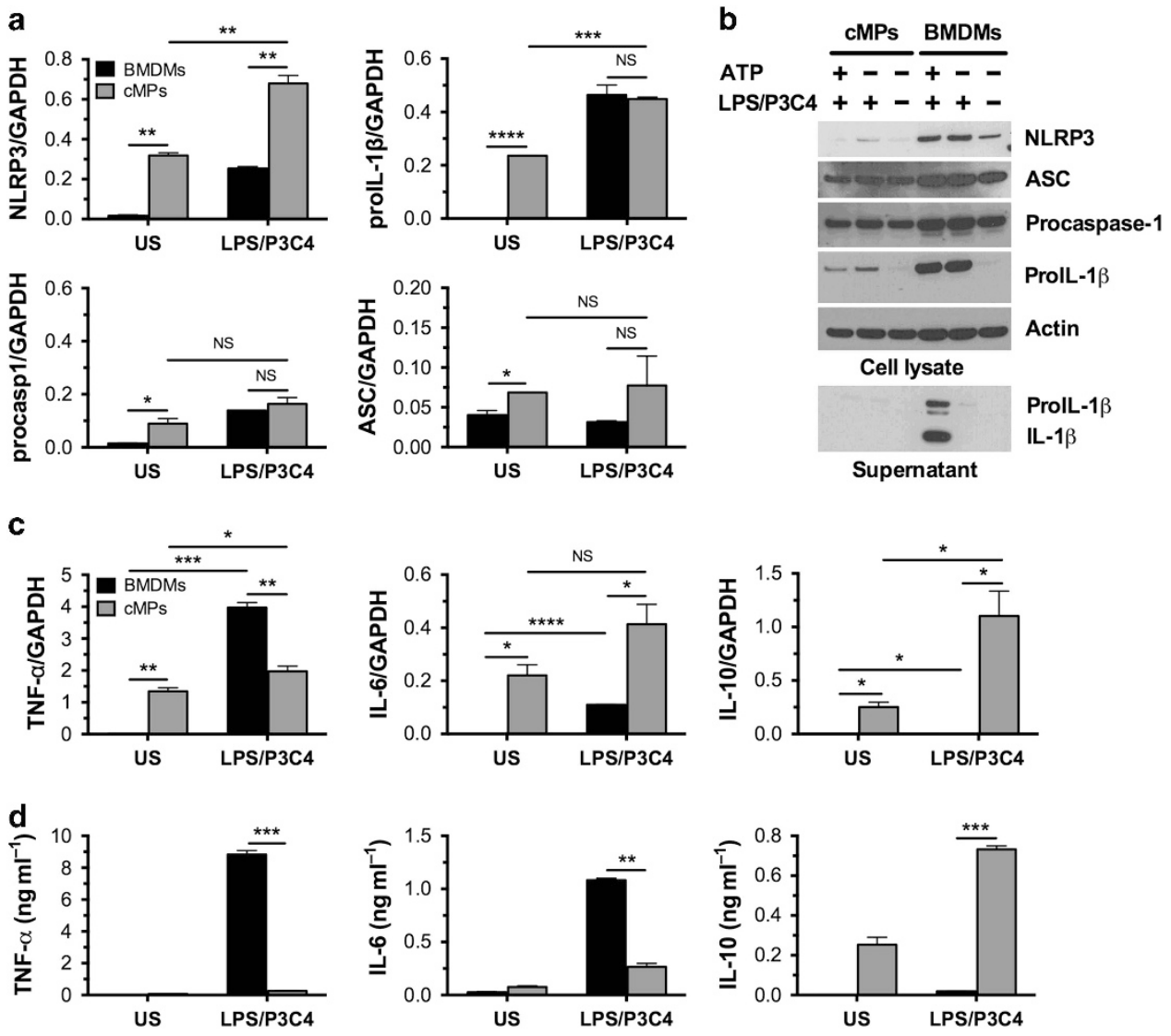

Figure 2 mRNA and protein levels for inflammasome components. Resident colonic macrophages (cMPs) and bone marrow-derived macrophages (BMDMs) were treated with lipopolysaccharide (LPS; $200 \mathrm{ng} \mathrm{ml}^{-1}$ ) and P3C4 $\left(2 \mu \mathrm{g} \mathrm{ml}^{-1}\right)$ for $4 \mathrm{~h}$ followed by ATP (5 mM) stimulation for $1 \mathrm{~h}$. (a) mRNA levels for NLRP3 (nucleotide-binding leucine-rich repeat-containing pyrin receptor 3), prolL-1 $\beta$, procaspase-1, and ASC. (b) Immunoblots of cell lysates and supernatants. Data are from sorted cells pooled from 90 colons. (c) mRNA levels and (d) protein measured in culture supernatants for tumor necrosis factor (TNF)- $\alpha$, interleukin (IL)-6, and IL-10. For $\mathbf{a}, \mathbf{c}$ and $\mathbf{d}$, data are from sorted cells pooled from 30 colons and are presented as the mean \pm s.d. of two biological replicates analyzed using an unpaired, two-tailed Student's $t$-test, ${ }^{*}<0.05$; ${ }^{* \star} P<0.01 ;{ }^{* \star \star} P<0.001$; NS, statistically not significant, $P>0.05$. For $\mathbf{b}$, data are from sorted cells pooled from 90 colons. All experiments shown are representative of at least three independent experiments with similar results. GAPDH, glyceraldehyde 3-phosphate dehydrogenase; procasp1, procaspase-1; US, unstimulated.

inflammasome activity in resident cMPs was not simply due to low levels of gene transcription following TLR signaling.

Importantly, however, despite very high levels of mRNA for proIL-1 $\beta$ and NLRP3, protein levels were low or undetectable in resident cMPs either before or after TLR stimulation (Figure 2b). This finding contrasted dramatically with BMDMs for which both mRNA and protein levels of NLRP3 and proIL-1 $\beta$ were significantly induced by TLR activation. Furthermore, tumor necrosis factor- $\alpha$ (TNF- $\alpha$ ) and IL-6 were regulated in a similar manner to NLRP3 and proIL- $1 \beta$ in cMPs, but in contrast, IL-10 was expressed in high levels for both mRNA and protein in resident cMPs before and after TLR stimulation compared with BMDMs (Figure 2c,d). Resident cMPs and BMDMs expressed similar levels of procaspase- 1 and ASC proteins.

These data strongly suggested that NLRP3 inflammasome activity in cMPs is controlled by posttranscriptional mechanisms that limit the accumulation of NLRP3 and proIL-1 $\beta$ protein in steady-state conditions. The low expression of proIL-1 $\beta$ observed in cMPs could explain why NLRC4 stimulation did not result in high levels of IL-1 $\beta$ release.
Our results also demonstrated that in a relevant tissue-specific cell type posttranscriptional control could apply to the regulation of TNF- $\alpha$, and possibly IL-6 (ref. 18-21) expression with gene specificity, as there was less effect on IL-10, procaspase-1, and ASC.

\section{cMPs isolated from mice with colitis are responsive to NLRP3 inflammasome activation}

The intestinal lamina propria macrophage compartment is dependent on continuous recruitment of $\mathrm{Ly}_{6} \mathrm{C}^{+}$ monocytes. ${ }^{1-4}$ As blood monocytes are fully responsive to NLRP3 activation, ${ }^{22}$ the maturation of unresponsive resident cMPs is likely driven by the factors in the intestinal microenvironment in the steady state, and such conditioning may be altered during inflammation. To address this question, we evaluated cMPs in RAG-deficient mice with colitis following adoptive transfer of naive $\mathrm{CD} 4{ }^{+} \mathrm{CD} 45 \mathrm{RB}{ }^{\mathrm{hi}} \mathrm{CD} 25^{-} \mathrm{T}$ cells. ${ }^{23}$ In contrast to resident cMPs from uninflamed mice, MHCII ${ }^{\mathrm{hi}} \mathrm{F} 4 / 80^{+} \mathrm{CD} 4^{+}$inflammatory monocytes and cMPs (infl. mono./cMPs) from mice with colitis secreted high levels of mature IL-1 $\beta$ after NLRP3 or NLRC4 activation (Figure 3a), 

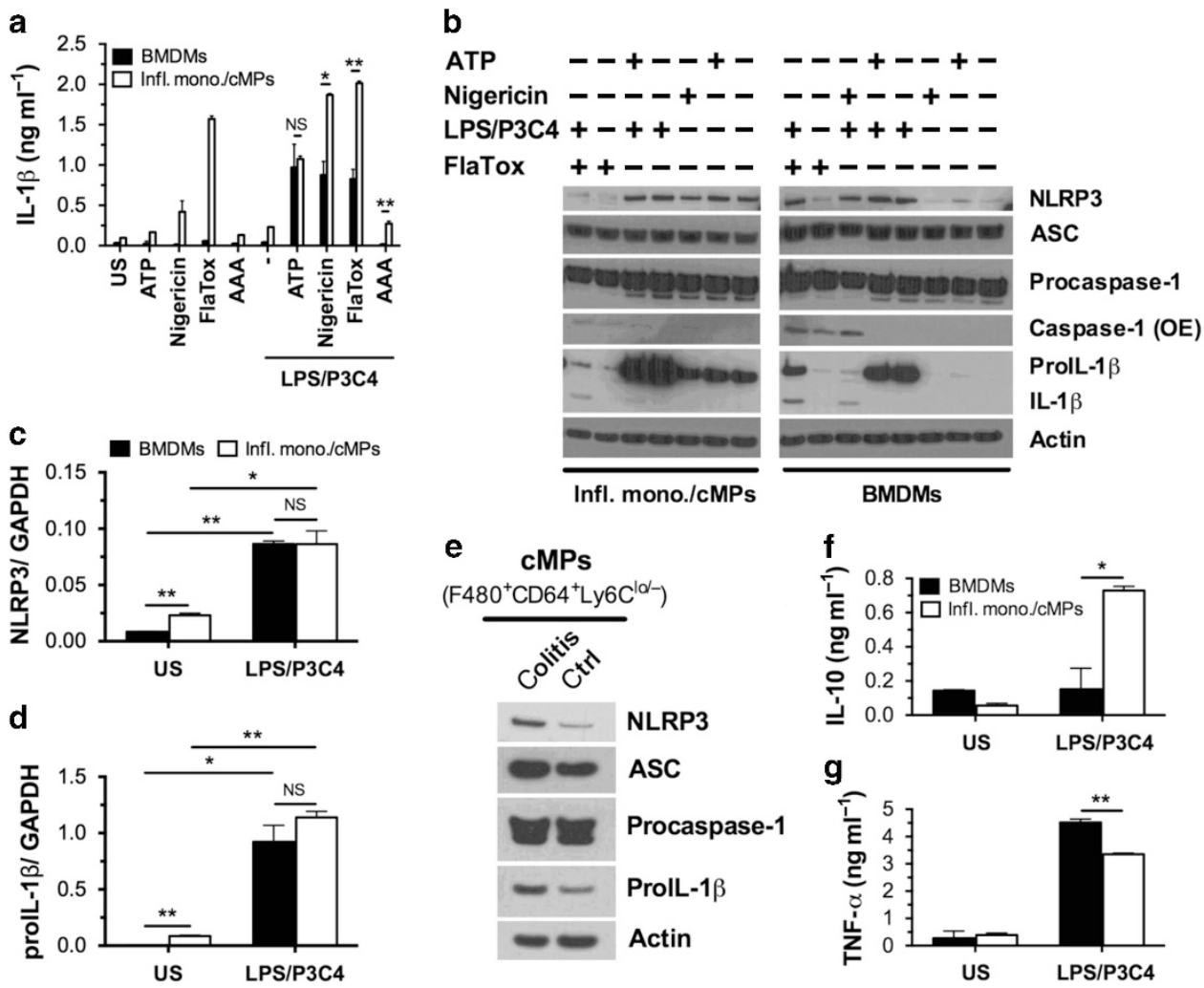

Figure 3 Inflammasome regulation in intestinal myeloid cells from mice with colitis. bone marrow-derived macrophages (BMDMs), inflammatory

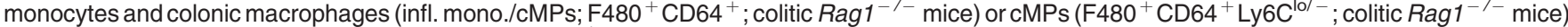

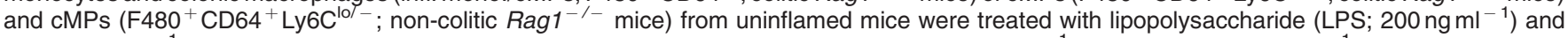
P3C4 $\left(2 \mu \mathrm{g} \mathrm{ml}^{-1}\right)$ for $4 \mathrm{~h}$ with or without stimulation with ATP $(5 \mathrm{~mm})$, nigericin $(10 \mu \mathrm{M})$, FlaTox $\left(3 \mu \mathrm{g} \mathrm{ml}^{-1}\right.$ of LFn-FlaA and $6 \mu \mathrm{g} \mathrm{ml}{ }^{-1}$ of protective antigen (PA)), or mutated FlaTox-negative control protein (AAA) $\left(3 \mu \mathrm{g} \mathrm{ml}^{-1}\right.$ of LFn-FlaAAA and $6 \mu \mathrm{g} \mathrm{ml}^{-1}$ of PA) for $1 \mathrm{~h}$. (a) Interleukin (IL)-1 $\beta$ measured in culture supernatants. (b) Immunoblot for NLRP3 (nucleotide-binding leucine-rich repeat-containing pyrin receptor 3 ) inflammasome components performed on cell lysates. (c) mRNA levels for NLRP3 and (d) prolL-1 $\beta$ from BMDMs and infl. mono./cMPs. (e) Immunoblot of inflammasome components from $\mathrm{F} 480^{+} \mathrm{CD}_{64}{ }^{+}$Ly6C $\mathrm{C}^{\mathrm{lo}}-\mathrm{CMPs}$ from uninflamed and inflamed Rag $1^{-/-}$mice. (f) IL-10 and (g) tumor necrosis factor (TNF)- $\alpha$ protein in culture supernatants from infl. mono./cMPs and BMDMs. Results for $\mathbf{a}, \mathbf{c}, \mathbf{d}, \mathbf{f}$, and $\mathbf{g}$ are from sorted cells pooled from 10 to 20 (infl. mono./cMPs) or 30 (cMPs) colons and are presented as the mean \pm s.d. of two biological replicates analyzed using an unpaired, two-tailed Student's $t$-test. ${ }^{\star} P<0.05 ;{ }^{\star \star} P<0.01 ; \mathrm{NS}$, statistically not significant, $P>0.05$. Results for $b$ are from sorted cells pooled from 160 or for e from 30 to 40 mice. All the data are representative of at least three independent experiments with similar results. GAPDH, glyceraldehyde 3-phosphate dehydrogenase; OE, overexposed; US, unstimulated.

consistent with their constitutive and TLR-inducible expression of NLRP3 and proIL-1 $\beta$ protein (Figure $3 \mathbf{b}$ ). In addition, similar to resident cMPs, infl. mono./cMPs expressed higher levels of NLRP3 and proIL-1 $\beta$ mRNA than BMDMs under resting condition but expressed much higher proIL-1 $\beta$ protein expression than resident cMPs (Figure 3c,d and see Supplementary Figure S2a). Furthermore, $\mathrm{MHCII}^{\mathrm{hi}} \mathrm{F} 4 /$ $80^{+} \mathrm{CD} 64^{+} \mathrm{Ly}_{6 \mathrm{C}} \mathrm{lo}^{-}-\mathrm{cMPs}$ population that lack $\mathrm{Ly}_{6 \mathrm{C}}{ }^{+}$ inflammatory monocytes (see Supplementary Figure S2b) exhibited the same enhancement in NLRP3 and proIL-1 $\beta$ protein expression in colitic mice as seen with total MHCII ${ }^{\mathrm{hi}} \mathrm{F} 4 / 80^{+} \mathrm{CD}^{+} 4^{+}$cells (Figure 3e), indicating that monocytes that mature into cMPs in the setting of inflammation retain enhanced inflammasome activity. Of note, immunoblots of cMPs from non-colitic RAG-deficient mice revealed a discrete proIL-1 $\beta$ band (Figure $3 \mathbf{e}$ ) that was undetectable in cMPs from wild-type (WT) B6 mice (Figure 1b and see Supplementary Figure 2a). The reason for this discrepancy is not yet clear; however, it is possible that B cells or regulatory $\mathrm{T}$ cells may contribute to posttranscriptional control, which is lacking in non-colitic RAG-deficient mice. Despite the discrepancy in baseline proIL-1 $\beta$ levels, a major difference in the levels of proIL-1 $\beta$ was still present between cMPs from colitic and non-colitic mice. Finally, although infl. mono./cMPs retain the ability to secrete high levels of the antiinflammatory cytokine IL-10 (Figure 3f), they produced robust levels of TNF- $\alpha$ (Figure 3g), suggesting a more general lack of posttranscriptional control of pro-inflammatory cytokine production by monocyte-derived cells under inflammatory conditions.

\section{NLRP3 and prolL-1 $\beta$ protein expression is regulated by proteasomal degradation in resident cMPs}

Both NLRP3 and proIL-1 $\beta$ may be subject to a number of posttranscriptional regulatory mechanisms. ${ }^{24}$ These include the regulation of mRNA splicing, polyadenylation, and stability, as well as control of protein translation and degradation. Recent reports have shown that the functional activity of NLRP3 can be regulated by ubiquitination/deubiquitination and that IL-1 $\beta$ protein levels may be controlled by 
a

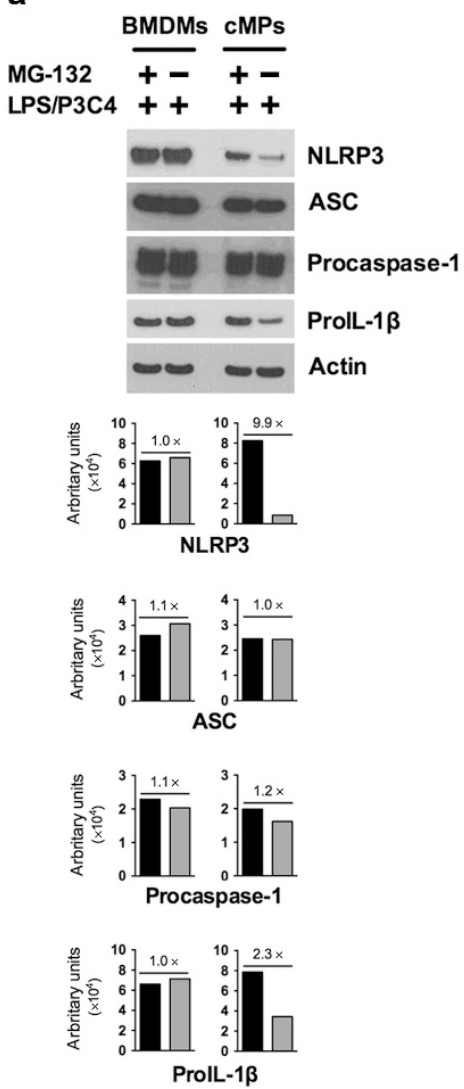

b

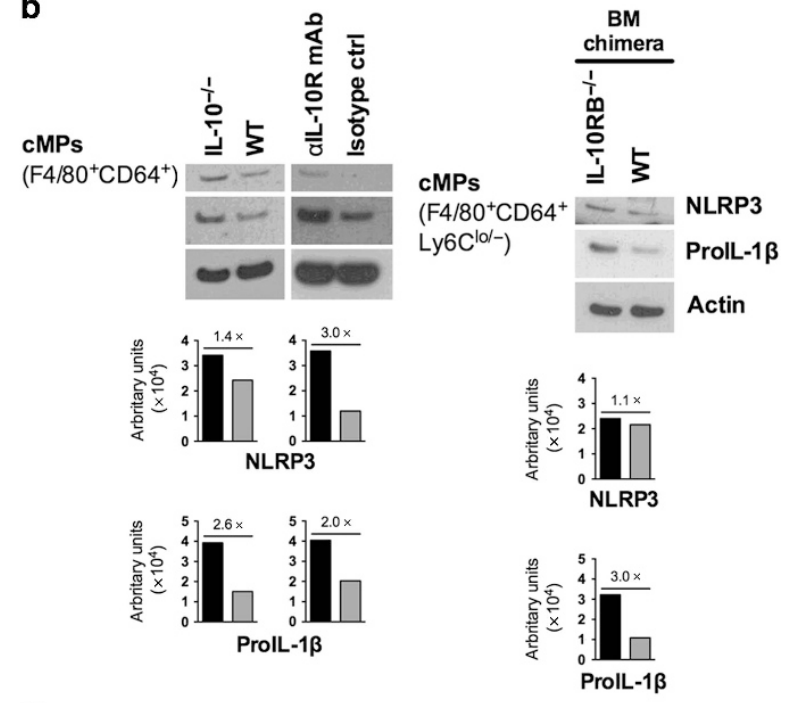

C
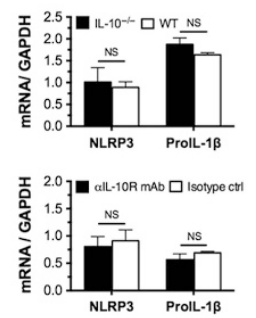

Figure 4 Regulation of NLRP3 (nucleotide-binding leucine-rich repeat-containing pyrin receptor 3 ) and prolL-1 $\beta$ protein expression by proteasomal degradation and interleukin (IL)-10. (a) Resident colonic macrophages (cMPs) from wild-type (WT) mice and bone marrow-derived macrophages (BMDMs) were pretreated with lipopolysaccharide (LPS; $200 \mathrm{ng} \mathrm{ml}^{-1}$ ) and P3C4 $\left(2 \mu \mathrm{g} \mathrm{ml}^{-1}\right)$ for $3 \mathrm{~h}$ followed by $1 \mathrm{~h}$ of treatment with the proteasome inhibitor MG-132 (5 $\mu \mathrm{m})$ or an equivalent volume of dimethyl sulfoxide. An immunoblot of cell lysates for NLRP3, ASC, procaspase-1, and prolL-1 $\beta$ is shown on the top, and densitometry data are shown at the bottom. Data are expressed in arbitrary units, as well as fold induction, of each experiment with sorted cells pooled from 60 colons for each experiment. Representative data of three independent experiments showing similar results. (b) Immunobotting for NLRP3 and prolL-1 $\beta$ protein was performed on cell lysates from $\mathrm{F} 4 / 80^{+} / \mathrm{CD} 64^{+} \mathrm{cMPs}$ from IL- $10^{-1-}$ or WT mice (left panel) or from WT mice treated with anti-IL-10R antibodies or isotype control for 11 days (middle panel) and from F4/80 ${ }^{+} / \mathrm{CD} 64^{+} \mathrm{Ly} \mathrm{C} \mathrm{C}^{\mathrm{lo} /-} \mathrm{cMPs}$ from WT/IL-10RB ${ }^{-/-}$ mixed chimeric mice (right panel). (c) mRNA levels for NLRP3 and prolL-1 $\beta$ from cMPs from IL-10 $0^{-1-}$ or WT mice (upper panel) or from WT mice treated with anti-IL-10R antibodies or isotype control for 11 days (bottom panel). Data are from a single experiment with sorted cells pooled from 30 colons from IL$10^{-1-}$ or WT mice, and representative data of two independent experiments showing similar results with sorted cells pooled from 30 colons from anti-IL10R or WT mice or 100 colons from mixed BM chimeric mice are shown. $P>0.05$. GAPDH, glyceraldehyde 3-phosphate dehydrogenase; NS, statistically not significant.

ubiquitination and proteasomal degradation in bone marrowderived dendritic cells in vitro. ${ }^{25-28}$ We first examined whether the low protein levels of NLRP3 and proIL1 $\beta$ in resident cMPs at steady state were due to rapid degradation by the proteasome. cMPs and BMDMs were treated with MG-132, a specific and efficient proteasome inhibitor. ${ }^{29-32}$ As MG-132 will suppress nuclear factor- $\kappa \mathrm{B}$ activation that is dependent on proteasomal degradation of I $\mathrm{B}$, cMPs and BMDMs were stimulated with LPS/P3C4 for $3 \mathrm{~h}$ prior to the addition of MG-132 for $1 \mathrm{~h}$. Blocking proteasome activity with MG-132 significantly increased the protein levels of NLRP 3 and proIL- $1 \beta$ in resident cMPs but not in BMDMs while neither ASC nor procaspase- 1 protein expression was affected in either cell population (Figure 4a). Similarly, cMPs treated with MG-132 in the absence of LPS/P3C4 pretreatment produced higher levels of NLRP3 and proIL-1 $\beta$ (see Supplementary Figure S4). These data are the first to indicate that NLRP3 levels may be controlled via the ubiquitin-proteasome system and show proteasome-dependent suppression of IL-1 $\beta$ production in a relevant tissue-derived cell population.

\section{IL-10 has a role in regulating NLRP3 and prolL-1 $\beta$ protein expression in resident cMPs}

The role of IL-10 signaling in maintaining immune homeostasis in the intestinal mucosa through the down-regulation of pro-inflammatory responses to bacterial products is well established. ${ }^{5,6,33,34}$ Therefore, we explored whether IL-10 signaling in resident cMPs could contribute to the posttranscriptional control of NLRP 3 and proIL-1 $\beta$ protein expression levels in resident cMPs. MHCII ${ }^{\mathrm{hi}} \mathrm{F} 4 / 80^{+} \mathrm{CD} 64^{+}$cMPs sorted from IL- $10^{-1-}$ mice before the onset of colitis (as indicated by the low numbers of $\mathrm{Ly} 6 \mathrm{C}^{+}$inflammatory monocytes in their colon tissue (see Supplementary Figure S3a)) indeed had significantly higher levels of NLRP3 and proIL-1 $\beta$ protein (Figure $4 \mathbf{b}$, left panel), whereas their mRNA levels were not significantly elevated in comparison to cMPs from IL-10-sufficient WT 
control mice (Figure 4c). These results were confirmed with cMPs from mice that received neutralizing anti-IL-10R antibodies for 11 days (see Supplementary Figure S3b, Figure $4 \mathbf{b}$ (middle panel), and Figure $4 \mathbf{c}$ ). To further rule out the possibility that underlying inflammation in mice lacking IL-10 or IL-10 receptor (IL-10R) signaling was responsible for these findings, we generated mixed bone marrow chimeric mice (WT:IL-10RB ${ }^{-1-} 1: 1$ ) and analyzed the levels of proIL-1 $\beta$ and NLRP3 protein in IL-10RB ${ }^{-1-}$ MHCII ${ }^{\mathrm{hi}} \mathrm{F} 4 / 80^{+} \mathrm{CD}^{+} 4^{+} \mathrm{Ly} 6 \mathrm{C}^{\mathrm{lo} /}-$ resident cMPs (see Supplementary Figure S3c). Consistently, we found that IL-10 is involved in the posttranscriptional regulation of NLRP3 and proIL-1 $\beta$ protein expressions in resident cMPs (Figure $4 \mathbf{b}$, right panel), which is not dependent on the presence of inflammation. Collectively, these results suggest that IL-10 has a role in regulating inflammasome activation in resident cMPs at the posttranscriptional level; however, whether this effect on cMPs is direct or indirect is not yet clear.

\section{DISCUSSION}

The data presented here demonstrate for the first time that inflammasome activation and cytokine production by resident cMPs are controlled by posttranscriptional mechanisms that are highly selective for certain genes and dependent on specific local environmental conditions in the intestine. Posttranscriptional control of NLRP3 and proIL- $1 \beta$, in addition to TNF- $\alpha$ and IL-6, in resident cMPs may contribute to the maintenance of immune homeostasis in the colon in steady state, as the activation of the NLRP3 inflammasome by microbial products (including LPS and ATP) induces not only the secretion of the pro-inflammatory cytokines IL-1 $\beta$ and IL-18 but also pyroptosis, a pro-inflammatory cell death program, uniquely dependent on caspase- 1 . We hypothesize that posttranscriptional regulation of NLRP3, proIL-1 $\beta$, TNF- $\alpha$, and IL- 6 in resident cMPs represents a mechanism to maintain intestinal homeostasis during constant exposure to commensal microbiota, yet poise these cells for rapid inflammatory cytokine production from high internal stores of mRNA in response to invading pathogens through as yet unidentified signals. Furthermore, while it is still unknown if resident cMPs are plastic and change their phenotype during initial stages of colitis induction, the current data also indicate that cMPs derived from circulating monocytes recruited during active inflammation have lost their capacity to regulate NLRP3 and proIL- $1 \beta$, as well as TNF- $\alpha$ and IL- 6 , through posttranscriptional control that likely contributes to chronic inflammation.

The release of IL- $1 \beta$ was impaired after NLRP3 inflammasome activation in highly purified resident cMPs. Although resident cMPs expressed high levels of mRNA for all NLRP3 inflammasome components, they expressed low-to-undetectable levels of proIL1 $\beta$ and NLRP3. In addition to the lack of the NLRP3 substrate proIL-1 $\beta$, stimulation of NLRP3 failed to result in the activation of caspase-1 in cMPs, indicating upstream effects on inflammasome activation that were most likely due to the low expression of NLRP3. Low levels of proIL-1 $\beta$ may also explain the low IL- $1 \beta$ release following
NLRC4 activation in cMPs. Whether NLRC4 protein levels are also posttranscriptionally regulated is not yet clear, as we were unable to detect NLRC4 protein by immunoblots with currently available antibodies. Furthermore, our data using highly purified cMPs are not necessarily inconsistent with a previous report showing lack of signaling through NLRP3 but not NLRC4 in intestinal $\mathrm{CD}_{11} \mathrm{~b}^{+}$phagocytes, which may facilitate the discrimination of commensal and pathogenic bacteria. ${ }^{35}$ The $\mathrm{CD} 11 \mathrm{~b}^{+}$population studied in this report contained $20-30 \% \mathrm{~F} 4 / 80^{-}$cells that may include dendritic cells and IgA ${ }^{+}$plasma cells, ${ }^{36}$ which may maintain NLRC4 activity in the intestinal environment. Evaluation of other cell populations will be important in future studies.

One important conclusion from the current data is that levels of mRNA in cMPs (and possibly other intestinal cell types) do not correlate with protein expression for a number of cytokines thought to be essential for intestinal inflammation. These data are important for the design of future studies and also indicate a need for reinterpreting prior experiments with rare tissue cells that relied on mRNA analysis as the only measure of gene expression and on which new hypotheses and research questions have been based.

The current findings also indicate for the first time that in addition to proIL-1 $\beta$, NLRP3 levels can be controlled via the ubiquitin-proteasome system. Recent reports have shown that both proIL-1 $\beta$ and NLRP3 are polyubiquitinated in primary cells in vitro but that ubiquitination results in several possible outcomes. Thus, following in vitro activation of murine bone marrow-derived dendritic cells and BMDMs with TLR ligands, proIL-1 $\beta$, after initial induction, was polyubiquitinated and progressive degraded over time, possibly as a mechanism to control its release in the setting of continued inflammasome activation. ${ }^{25}$ In contrast, others have shown that, in BMDMs, proIL- $1 \beta$ is constitutively ubiquitinated (likely by the addition of K63-linked and unanchored polyubiquitin to the proIL1 $\beta$ complex), which enhances proIL-1 $\beta$ interaction with procaspase-1, and thus increasing NLRP3 activity in these cells. ${ }^{28}$ Furthermore, it has also been reported that in BMDMs constitutive polyubiqutination of NLRP3 inhibits its activity (rather than by inducing its degradation) and that deubiquitination following TLR or ATP activation results in enhanced activation. ${ }^{26,27}$ In the present study, we demonstrated that the inhibition of proteasome activity by MG-132 significantly increased the protein levels of both NLRP 3 and proIL-1 $\beta$ in resident cMPs but displayed no effect in BMDMs. Because the effects of MG-132 on the proteasome are highly specific, ${ }^{29-32}$ and MG-132 is now accepted as a highly effective way to block proteasome activity, we believe the current data are the first to indicate the role of the ubiquitin/proteasome system in the degradation of both NLRP3 and proIL- $1 \beta$ as a mechanism to control inflammasome activation in vivo, and in a relevant tissue-specific cell population, the intestinal macrophage. Although a direct demonstration of NLRP3 and proIL-1 $\beta$ ubiquitination in cMPs by immunoprecipitation and immunoblotting would affirm these conclusions, these experiments are practically impossible, as they would require purified 
cMPs from an estimated 500 mice per experiment. Therefore, we are currently exploring the possibility of conditioning BMDMs or cell lines in vitro to mimic our findings in cMPs to allow for further confirmation of direct ubiquitination.

IL-10-signaling of cMPs likely has an important role in maintaining immune homeostasis in the intestine as deletion of the IL-10R in CX3CR $1^{+}$or lysozyme-expressing cells that include resident cMPs results in pro-inflammatory responses to bacterial products and spontaneous colitis, ${ }^{5,6}$ similar to IL-10Rdeficient humans with severe intestinal bowel diseases. ${ }^{33,34}$ IL-10 and downstream IL-10R signaling are important to control the activation of mucosal T cells, ${ }^{37-39}$ as well as innate cells such as dendritic cells and macrophages. ${ }^{5}$ Furthermore, cMPs are a major source of IL-10 in the intestine, ${ }^{5}$ and IL-10 production does not appear to be highly controlled by posttranscriptional mechanisms, as shown here. Therefore, we determined whether IL-10 may be involved in posttranscriptional control of inflammatory cytokines in vivo. We found that resident cMPs isolated from IL- $10^{-1-}$ mice, or mice treated with neutralizing anti-IL-10R antibodies, before the onset of colitis had significantly increased the expression of NLRP3 and proIL- $1 \beta$ protein in comparison to cMPs from untreated WT mice. As the absence of IL-10 in the intestinal environment can cause inflammation and the recruitment of inflammatory monocytes to the intestinal tissue, ${ }^{5,33,40}$ which are fully responsive to NLRP3 activation, ${ }^{22}$ we confirmed these findings in resident cMPs $\left(\mathrm{Ly} 6 \mathrm{C}^{\mathrm{lo} /-}\right)$ from mixed IL$10 \mathrm{RB}^{-1-} \times \mathrm{WT}$ bone marrow chimeric mice without inflammation. Together these results indicated that IL-10 contributes to the posttranscriptional regulation of NLRP 3 and proIL- $1 \beta$ protein expression in resident cMPs. However, whether IL-10 acts directly on cMPs to control NLRP3 and proIL-1 $\beta$ expression or indirectly through effects on other cell types remains unclear, as experiments to address this issue are currently impractical owing to high costs (e.g., IL-10 $10^{-/-}$mice to perform IL-10 add-back experiments on purified cMPs) or poor specificity of conditional knockout mice for cMPs. Furthermore, given that the protein levels seen in IL-10 signaling-deficient cells were still low compared with levels seen in TLR-stimulated BMDMs with a similar level of mRNA, additional environmental factors are likely involved.

At present, we have no evidence for additional posttranscriptional control mechanisms for NLRP3 or proIL-1 $\beta$, and have not yet addressed the mechanisms regulating TNF- $\alpha$ or IL- 6 in resident cMPs. For example, we have no definitive evidence for splice variants of NLRP3, proIL- $\beta$, or other cytokines, and as levels of mRNA are high, increased mRNA degradation does not appear to account for low protein levels. In this regard, miR223, which can suppress NLRP3 expression and activation in $\mathrm{BMDMs}^{41}$ by inhibiting mRNA accumulation, is not likely involved in the current effects, despite its high levels in cMPs (data not shown). Inhibition of translation could certainly contribute to the poor protein production seen in the present studies, in particular for TNF- $\alpha$, for which IL-10 was shown in in vitro studies of thioglycolate-elicited pMPs and BMDMs to block both transcription and translation, but not mRNA stability. ${ }^{18,19}$ Unfortunately, techniques to analyze mRNA translation for specific genes by ribosome profiling, microarray analysis of mRNA associated with polysomes, or translational profiling through the affinity purification of epitope-tagged ribosomes cannot be performed on the small number of cMPs that we can isolate, so at the present time there is no clear method to determine translational efficiencies for NLRP 3 and proIL- $1 \beta$ by resident cMPs. Interestingly, IL-6 has been shown to be highly regulated through effects on mRNA stability, but this does not seem to have a primary role in resident cMPs for which IL-6 mRNA levels are high. ${ }^{20,21}$ Future studies will need to address these issues.

In conclusion, our data demonstrate that NLRP3 inflammasome activation and pro-inflammatory cytokine production by resident cMPs are controlled by posttranscriptional mechanisms that are highly selective for certain genes and dependent on local environmental conditions. These mechanisms likely contribute to immune homeostasis in a constantly challenged intestinal environment but are lost during intestinal inflammation. Although multiple posttranscriptional mechanisms may be involved, our data indicate that the degradation of NLRP3 and proIL- $1 \beta$ proteins by the ubiquitin-proteasome pathway is one such mechanism and that IL-10 has a role in steady-state conditioning. Further characterization of posttranscriptional control mechanisms in cMPs should contribute to our understanding of intestinal pathophysiology and may provide new targets for the treatment of patients with intestinal bowel diseases.

\section{METHODS}

Antibodies and reagents. Ultrapure Escherichia coli LPS (K12 strain) and synthetic bacterial lipoprotein Pam3Cys-Ser-(Lys)4 (P3C4) were obtained from Invivogen (San Diego, CA); ATP and nigericin from Sigma-Aldrich (St Louis, MO). FlaTox, an engineered protein complex containing components of Bacillus anthracis toxin (amino-terminal domain of $B$. anthracis lethal factor and anthrax protective antigen channel) that allows cytosolic delivery of Legionella pneumophila flagellin or its mutated inactive form (AAA), ${ }^{42}$ was a gift from $\mathrm{Dr}$ Russell Vance (University of California, Berkeley, CA). Anti-IL-10R monoclonal antibody (clone 1B1.3a) was a kind gift from David Sacks, NIAID, NIH, Bethesda, MD. Control rat IgG was obtained from BioXCell (West Lebanon, NH). The proteasome inhibitor MG-132 was from Enzo Life Sciences (Farmingdale, NY). Antibodies used in the study were the following: anti-NLRP3/NALP3 mAb (Cryo-2) was obtained from AdipoGen (San Diego, CA); anti-IL-1 $\beta$ from R\&D Systems (Minneapolis, MN); anti-ASC (N-15) and anti-caspase-1 p10 (M-20) from Santa Cruz Biotechnology (Dallas, TX); anti-actin from Cell Signaling Technology (Danvers, MA); anti-mouse horseradish peroxidase (HRP), anti-rat IgG HRP, anti-rabbit IgG HRP, and antigoat IgG HRP from Jackson ImmunoResearch Laboratories, (West Grove, PA); Fc block/anti-CD16/32 (2.4G2) and directly conjugated monoclonal antibodies (mAb/clone): anti-CD19/eBio1D3, B220/RA3-6B2, TCR- $\beta / H 57-597$, TCR- $\gamma \delta /$ BBioGL3, MHC II/M5114.15.2, Ly6C/HK1.4, CD4/GL1.5, CD45RB/M1-70, CD25/PC61.5, CD45.1/A20, and CD45.2/104 used in flow cytometry were from eBioscience (San Diego, CA). Other conjugated mAb (anti-F4/80/BM8 and CD64/X54-5/7.1) were from Biolegend (San Diego, CA).

Mice. All the experiments were performed using C57BL/6 (B6) mice. B6 mice were obtained from the National Cancer Institute and used at 
6-12 weeks of age. Rag1 ${ }^{-/-}$mice were purchased from Taconic Farms (Derwood, MD). IL-10 $0^{-1-}$ and IL-10RB ${ }^{-1-}$ mice were purchased from The Jackson Laboratory (Sacramento, CA). All mice were maintained at an American Association for the Accreditation of Laboratory Animal Care-accredited animal facility at the National Institute of Allergy and Infectious Diseases and housed in accordance with the procedures outlined in the Guide for the Care and Use of Laboratory Animals under an animal study proposal approved by the National Institute of Allergy and Infectious Diseases Animal Care and Use Committee.

Experimental animal models. $T$ cell transfer colitis. Total $\mathrm{CD} 4{ }^{+}$ T cells were isolated from the spleens of 6-8-week-old female B6 mice via negative selection using the magnetic activation cell sorter CD4 T-cell Isolation Kit (Miltenyi Biotec, Auburn, CA) according to the manufacturer's instructions. Enriched cells were subsequently sorted for naive $\mathrm{CD}^{+}{ }^{+} \mathrm{CD} 25^{-} \mathrm{CD} 45 \mathrm{RB}^{\text {high }} \mathrm{T}$ cells using FACS (FACSAria, BD Biosciences, San Jose, CA). In all, $3 \times 10^{5} \mathrm{CD}^{+} \mathrm{CD} 25$ $\mathrm{CD} 45 \mathrm{RB}^{\text {high }}$ cells were transferred intraperitoneally to recipient 6-8week-old female $\mathrm{Rag}^{-1-}$ mice, which were subsequently housed under specific pathogen-free non-sterile conditions (nonsterile bedding, nonsterile food, and non-acidified water). ${ }^{23}$ The development of intestinal inflammation was monitored by weight loss, which correlates with histological evidence of colitis and increased cell numbers of MHC II $^{\text {hi }}$ cells in the colon. The study end point was defined as a mean weight loss of $25-30 \%$ from initial weight of

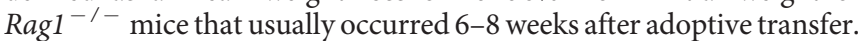
For each analysis, cells were collected and pooled from 20 to 30 animals per experiment.

Neutralization of $I L-10$ receptor in vivo. WT B6 mice were intraperitoneally treated with three doses of $0.5 \mathrm{mg}$ per mice of anti-IL-10R monoclonal antibody (1B1.3a) or isotype control/ phosphate-buffered saline for 11 days. After the treatment, mice were euthanized and the colons were removed to obtain purified and FACS-sorted cMPs as described bellow.

$I L-10 R B^{-/-} / W T$ mixed bone marrow chimeric mice. Mixed chimeric mice were generated by reconstituting lethally irradiated (900 rads) WT B6 mice with $3 \times 10^{6}$ total bone marrow cells intravenously isolated from both WT (CD45.1) and IL-10RB ${ }^{-1-}$ (CD45.2) mice (1:1 ratio). B6 bone marrow chimeric mice received drinking water containing antibiotics $\left(0.13 \mathrm{mg} \mathrm{ml}^{-1}\right.$ Trimethoprim and $0.67 \mathrm{mg} \mathrm{ml}^{-1}$ Sulfamethoxazole) for 4 weeks, followed by 4 weeks of regular water. Similar percentages of cMPs from chimeric mice were found to be CD45.1 and CD45.2, indicating equivalent engraftment and development of WT and IL-10RB ${ }^{-1-}$ cMPs.

Macrophage isolation, differentiation, and activation. cMPs were prepared as described previously. ${ }^{2}$ Briefly, entire colons from 30 to 50 mice were collected and washed with calcium and magnesium-free Hanks' balanced salt solution (HBSS; Gibco; Grand Island, NY). The tissues were opened longitudinally, cut into $2-\mathrm{cm}$ pieces, and incubated with HBSS containing $0.015 \%$ dithiothreitol (Sigma-Aldrich) for $15 \mathrm{~min}$, followed by extensive washing in cold HBSS containing 5\% heat-inactivated fetal bovine serum (GemCell, West Sacramento, CA) to remove epithelial cells and mucus. Colon pieces were finely minced and digested in complete (supplemented with penicillinstreptomycin, $2 \mathrm{~mm}$ L-glutamine, $15 \mathrm{~mm}$ HEPES (all from Gibco), $50 \mu \mathrm{M} \beta$-mercaptoethanol (Sigma-Aldrich), and $10 \%$ fetal bovine serum) Iscove's media (Gibco) containing $167 \mu \mathrm{g} \mathrm{ml}^{-1}$ Liberase TL and $30 \mu \mathrm{g} \mathrm{ml}^{-1} \mathrm{DNase} \mathrm{I}$ (Roche, Indianapolis, IN) for $60 \mathrm{~min}$ at $37^{\circ} \mathrm{C}$. The samples were filtered, pelleted, and resuspended in $1.077 \mathrm{~g} \mathrm{~cm}^{-3}$ Optiprep (Axis-Shield, Dundee, Scotland). After centrifugation at 2,000 r.p.m. for $15 \mathrm{~min}$ at room temperature, the viable cells from the layer surface were collected and cMPs were sorted using FACSAriaTM (BD Biosciences). To obtain cMPs, MHCII ${ }^{\text {hi }} \mathrm{F} 4 / 80^{\text {hi }} \mathrm{CD} 64^{+}$cells were sorted. Resident pMPs were obtained by washing the peritoneal cavity of B6 mice with cold HBSS, and the resulting cells were sorted for
CD19- ${ }^{-}$MHC II $^{\text {hi }}$ $4 / 80^{\text {hi }}$ cells. BMDMs were generated by flushing femurs and tibias with phosphate-buffered saline, and precursor cells were cultured in complete RPMI 1640 media (Gibco) supplemented with 30\% L929 cell-conditioned medium, as the source of macrophage colony-stimulating factor. After 7 days in culture, mature BMDMs were harvested using CellStripper (CellGro, Pittsburgh, PA).

Inflammasome activation and proteasome inhibition. Sorted cMPs, pMPs, or BMDMs in complete RPMI 1640 media were added to 96-well plates $\left(1 \times 10^{5}\right.$ cells per $\left.100 \mu \mathrm{l}\right)$ and stimulated with ultrapure LPS $\left(200 \mathrm{ng} \mathrm{ml}^{-1}\right)$ and P3C4 $\left(2 \mu \mathrm{g} \mathrm{ml}^{-1}\right)$ for $4 \mathrm{~h}$ at $37^{\circ} \mathrm{C}$, followed by ATP $(5 \mathrm{~mm})$ or nigericin $(10 \mu \mathrm{M})$ or FlaTox $\left(3 \mu \mathrm{g} \mathrm{ml}{ }^{-1}\right.$ of LFn-FlaA and $6 \mu \mathrm{g} \mathrm{ml}^{-1}$ of protective antigen) or mutated Flatox-negative control protein (AAA) $\left(3 \mu \mathrm{g} \mathrm{ml}^{-1}\right.$ of LFn-FlaAAA and $6 \mu \mathrm{g} \mathrm{ml}^{-1}$ of protective antigen) stimulation for $1 \mathrm{~h}$ at $37^{\circ} \mathrm{C}$. In some experiments, cMPs, BMDMs, or pMPs were stimulated with ultrapure LPS $\left(200 \mathrm{ng} \mathrm{ml}^{-1}\right)$ and P3C4 $\left(2 \mu \mathrm{g} \mathrm{ml}^{-1}\right)$ for $3 \mathrm{~h}$ at $37^{\circ} \mathrm{C}$, followed by proteasomal inhibitor MG-132 $(5 \mu \mathrm{M})$ or an equivalent volume of dimethyl sulfoxide (Sigma-Aldrich) treatment for $1 \mathrm{~h}$ at $37^{\circ} \mathrm{C}$.

Measurement of cytokines. Cell culture supernatants were harvested after $5 \mathrm{~h}$ of culture in medium alone or after treatment with LPS/P3C4 or LPS/P3C4 plus inflammasome activators. Mouse cytokines concentrations were measured by conventional double-sandwich enzymelinked immunosorbent assay kits from eBioscience (IL-1 $\beta$ and IL-6) and R\&D Systems (TNF- $\alpha$ and IL-10), respectively, according to the manufacturer's instructions.

RNA isolation and real-time reverse transcriptase-PCR. For realtime reverse transcriptase-PCR analysis of gene expression, cMPs and BMDMs were collected, and total RNA was isolated using an RNeasy Mini Kit (Qiagen, Valencia, CA) following the manufacturer's instructions. cDNA was synthetized using qScript cDNA Supermix (Quanta, Gaithersburg, MD) and real-time PCR analysis was performed on the 7900HT Fast Real-Time PCR instrument (Applied Biosystems, Grand Island, NY) using FAM-labeled gene-specific probes and primers (Applied Biosystems). The level of target gene expression was calculated as $2^{-\Delta \mathrm{Ct}}$ where $\Delta \mathrm{Ct}=\mathrm{Ct}_{\text {target }}-\mathrm{Ct}_{\mathrm{GAPDH}}$, with glyceraldehyde 3-phosphate dehydrogenase (GAPDH) as endogenous control and $\mathrm{Ct}$ indicating threshold cycle.

Immunoblotting. Cells were lysed in buffer containing $1 \%$ Triton-X (Sigma-Aldrich) supplemented with complete protease inhibitor cocktail (Cell Signaling). For the reduction of samples, NuPAGE sample reducing agent (Invitrogen, Grand Island, NY) was added. Supernatants were precipitated with methanol/chloroform before NLRP3 inflammasome components were analyzed. Lysates or supernatants were resolved by sodium dodecyl sulfate polyacrylamide gel electrophoresis (SDS-PAGE) using $4-12 \%$ Bis-Tris precast mini gels in an X-Cell Sure-Lock Mini-Cell (Novex; Invitrogen) following the manufacturer's instructions. SDS-PAGE gel proteins were transferred to nitrocellulose membranes by electro-blotting using an iBlot System (Life Technologies, Grand Island, NY). The membranes were immunoblotted with respective antibodies and developed using SuperSignal West Pico chemiluminescent substrate (Thermo Scientific, Waltham, MA). The NIH ImageJ software gel-analysis method was used to quantify immunoblotting bands. The background was subtracted, and the absolute density of each peak is shown as arbitrary units.

Statistical analysis. Except where indicated, all experiments shown are representative of three or more independent experiments with similar results. An unpaired, two-tailed Student's $t$-test was performed using the Prism 6 software (GraphPad Software, La Jolla, CA). Differences with a value of $P<0.05\left(^{*}\right), P<0.011^{(*)}$, or $P<0.0011^{(* *)}$ were considered significant.

SUPPLEMENTARY MATERIAL is linked to the online version of the paper at http://www.nature.com/mi 


\section{ACKNOWLEDGMENTS}

This work was supported by the Division of Intramural Research, NIAID, NIH. A.A.F. was a recipient of the Brazilian National Council for Scientific and Technological Development/Science Without Borders Program (CNPq/CsF) postdoctoral fellowship. We thank Alan Sher, Dan Barber, Dragana Jankovic, and Katrin Meyer-Barber for their helpful discussion, Elina Stregevsky from NIAID FACS sorting facility, and the members of the NIAID Comparative Medicine Branch animal facilities for technical assistance.

\section{DISCLOSURE}

The authors declared no conflict of interest.

(c) 2016 Society for Mucosal Immunology

\section{REFERENCES}

1. Bain, C.C. et al. Resident and pro-inflammatory macrophages in the colon represent alternative context-dependent fates of the same Ly6Chi monocyte precursors. Mucosal Immunol. 6, 498-510 (2013).

2. Rivollier, A., He, J., Kole, A., Valatas, V. \& Kelsall, B.L. Inflammation switches the differentiation program of Ly6Chi monocytes from antiinflammatory macrophages to inflammatory dendritic cells in the colon. J. Exp. Med. 209, 139-155 (2012).

3. Tamoutounour, S. et al. CD64 distinguishes macrophages from dendritic cells in the gut and reveals the Th1-inducing role of mesenteric lymph node macrophages during colitis. Eur. J. Immunol. 42, 3150-3166 (2012).

4. Zigmond, E. et al. Ly6C hi monocytes in the inflamed colon give rise to proinflammatory effector cells and migratory antigen-presenting cells. Immunity 37, 1076-1090 (2012).

5. Shouval, D.S. et al. Interleukin-10 receptor signaling in innate immune cells regulates mucosal immune tolerance and anti-inflammatory macrophage function. Immunity 40, 706-719 (2014).

6. Zigmond, E. et al. Macrophage-restricted interleukin-10 receptor deficiency, but not IL-10 deficiency, causes severe spontaneous colitis. Immunity 40, 720-733 (2014).

7. Lavin, $\mathrm{Y}$. et al. Tissue-resident macrophage enhancer landscapes are shaped by the local microenvironment. Cell 159, 1312-1326 (2014).

8. Franchi, L., Munoz-Planillo, R. \& Nunez, G. Sensing and reacting to microbes through the inflammasomes. Nat. Immunol. 13, 325-332 (2012).

9. Hu, B. et al. Inflammation-induced tumorigenesis in the colon is regulated by caspase-1 and NLRC4. Proc. Natl. Acad. Sci. USA 107, 21635-21640 (2010).

10. Bauer, C. et al. Colitis induced in mice with dextran sulfate sodium (DSS) is mediated by the NLRP3 inflammasome. Gut 59, 1192-1199 (2010).

11. Monteleone, G. et al. Bioactive IL-18 expression is up-regulated in Crohn's disease. J. Immunol. 163, 143-147 (1999).

12. Casini-Raggi, V. et al. Mucosal imbalance of $\mathrm{IL}-1$ and $\mathrm{IL}-1$ receptor antagonist in inflammatory bowel disease. A novel mechanism of chronic intestinal inflammation. J. Immunol. 154, 2434-2440 (1995).

13. Ligumsky, M., Simon, P.L., Karmeli, F. Rachmilewitz, D. Role of interleukin 1 in inflammatory bowel disease-enhanced production during active disease. Gut 31, 686-689 (1990).

14. Pizarro, T.T. et al. IL-18, a novel immunoregulatory cytokine, is up-regulated in Crohn's disease: expression and localization in intestinal mucosal cells. J Immunol 162, 6829-6835 (1999).

15. Denning, T.L., Wang, Y.C., Patel, S.R., Williams, I.R. \& Pulendran, B. Lamina propria macrophages and dendritic cells differentially induce regulatory and interleukin 17-producing Tcell responses. Nat. Immunol. 8, 1086-1094 (2007).

16. Smythies, L.E. et al. Human intestinal macrophages display profound inflammatory anergy despite avid phagocytic and bacteriocidal activity. J. Clin. Invest. 115, 66-75 (2005).

17. Bauernfeind, F.G. et al. Cutting edge: NF-kappaB activating pattern recognition and cytokine receptors license NLRP3 inflammasome activation by regulating NLRP3 expression. J. Immunol. 183, 787-791 (2009).
18. Kontoyiannis, D. et al. Interleukin-10 targets p38 MAPK to modulate ARE-dependent TNF mRNA translation and limit intestinal pathology. EMBO J. 20, 3760-3770 (2001).

19. Smallie, T. et al. IL-10 inhibits transcription elongation of the human TNF gene in primary macrophages. J. Exp. Med. 207, 2081-2088 (2010).

20. Iwasaki, $\mathrm{H}$. et al. The IkappaB kinase complex regulates the stability of cytokine-encoding mRNA induced by TLR-IL-1R by controlling degradation of regnase-1. Nat. Immunol. 12, 1167-1175 (2011).

21. Masuda, K. et al. Arid5a controls IL-6 mRNA stability, which contributes to elevation of IL-6 level in vivo. Proc. Natl. Acad. Sci. USA 110, 9409-9414 (2013).

22. Guarda, G. et al. Type I interferon inhibits interleukin-1 production and inflammasome activation. Immunity 34, 213-223 (2011).

23. Powrie, F., Leach, M.W., Mauze, S., Caddle, L.B. \& Coffman, R.L. Phenotypically distinct subsets of CD4 $+\mathrm{T}$ cells induce or protect from chronic intestinal inflammation in C. B-17 scid mice. Int. Immunol. 5, 1461-1471 (1993).

24. Carpenter, S., Ricci, E.P., Mercier, B.C., Moore, M.J. \& Fitzgerald, K.A. Post-transcriptional regulation of gene expression in innate immunity. Nat. Rev. Immunol. 14, 361-376 (2014).

25. Ainscough, J.S. et al. Dendritic cell IL-1alpha and IL-1beta are polyubiquitinated and degraded by the proteasome. J. Biol. Chem. 289, 35582-35592 (2014).

26. Juliana, C. et al. Non-transcriptional priming and deubiquitination regulate NLRP3 inflammasome activation. J. Biol. Chem. 287, 36617-36622 (2012).

27. Py, B.F., Kim, M.S., Vakifahmetoglu-Norberg, H. \& Yuan, J. Deubiquitination of NLRP3 by BRCC3 critically regulates inflammasome activity. Mol. Cell 49, 331-338 (2013).

28. Duong, B.H. et al. A20 restricts ubiquitination of pro-interleukin-1beta protein complexes and suppresses NLRP3 inflammasome activity. Immunity 42, 55-67 (2015).

29. Elliott, P.J., Zollner, T.M. \& Boehncke, W.H. Proteasome inhibition: a new anti-inflammatory strategy. J. Mol. Med. (Berl) 81, 235-245 (2003).

30. Kisselev, A.F. \& Goldberg, A.L. Proteasome inhibitors: from research tools to drug candidates. Chem. Biol. 8, 739-758 (2001).

31. Lee, D.H. \& Goldberg, A.L. Proteasome inhibitors: valuable new tools for cell biologists. Trends Cell. Biol. 8, 397-403 (1998).

32. Moore, B.S., Eustaquio, A.S. \& McGlinchey, R.P. Advances in and applications of proteasome inhibitors. Curr. Opin. Chem. Biol. 12, 434-440 (2008).

33. Glocker, E.O., Kotlarz, D., Klein, C., Shah, N. \& Grimbacher, B. IL-10 and IL-10 receptor defects in humans. Ann NY Acad. Sci. 1246, 102-107 (2011).

34. Murugan, D. et al. Very early onset inflammatory bowel disease associated with aberrant trafficking of IL-10R 1 and cure by T cell replete haploidentical bone marrow transplantation. J. Clin. Immunol. 34, 331-339 (2014).

35. Franchi, L. et al. NLRC4-driven production of IL-1beta discriminates between pathogenic and commensal bacteria and promotes host intestinal defense. Nat. Immunol. 13, 449-456 (2012).

36. Kunisawa, J. et al. Microbe-dependent CD11b + IgA + plasma cells mediate robust early-phase intestinal IgA responses in mice. Nat. Commun. 4, 1772 (2013).

37. Chaudhry, A. et al. Interleukin-10 signaling in regulatory T cells is required for suppression of Th17 cell-mediated inflammation. Immunity 34, 566578 (2011).

38. Murai, M. et al. Interleukin 10 acts on regulatory $\mathrm{T}$ cells to maintain expression of the transcription factor Foxp3 and suppressive function in mice with colitis. Nat. Immunol. 10, 1178-1184 (2009).

39. Huber, S. et al. Th17 cells express interleukin-10 receptor and are controlled by Foxp3(-) and Foxp3 + regulatory CD4 + T cells in an interleukin-10-dependent manner. Immunity 34, 554-565 (2011).

40. Glocker, E.O. et al. Inflammatory bowel disease and mutations affecting the interleukin-10 receptor. N. Engl. J. Med. 361, 2033-2045 (2009).

41. Bauernfeind, F. et al. NLRP3 inflammasome activity is negatively controlled by miR-223. J. Immunol. 189, 4175-4181 (2012).

42. von Moltke, J. et al. Rapid induction of inflammatory lipid mediators by the inflammasome in vivo. Nature 490, 107-111 (2012). 